\title{
Characterization of Structure and Self-Diffusion of Elastomers by NMR Method
}

\author{
T.P. Kulagina*, G.E. Karnaukh, L.P. Smirnov \\ Institute of Problems of Chemical Physics RAS, Chernogolovka, Moscow region, 142432, Russia
}

\begin{abstract}
In this work the theory of free induction decay in linear and cross-linked flexible polymers with a various average length of a polymeric chain in a wide temperature is represented. It is shown the change of topological structure of linear polymers with increasing of mean molecular weight $M_{w}$, and at $M_{w}>10^{5}$ the physical network of entanglements starts being formed. The correlation function of molecular motion which is applied to calculation of diffusion attenuation of spin echo is received. The general approach to calculation of signals of diffusion attenuation spin echo and its application for determination of coefficient of self-diffusion in elastomers with various topological structures is offered. It allowed explanation observed experimentally anomalous diffusion.
\end{abstract}

DOI: $10.12693 /$ APhysPolA.127.1128

PACS: $76.20+q, 76.60 . \mathrm{Lz}, 77.84 . J \mathrm{~d}$

\section{Introduction}

For carrying out operated synthesis and application of elastomers (El) and composites on their basis, data on their structure, molecular mobility and diffusion are necessary. The data are defined by different methods NMR. The success in quantitative definition of these characteristics in many respects depends on the accuracy of a choice of a technique of NMR and well developed theory, allowing to connect structural and dynamic parameters with observed signals of NMR [1-4].

Topological structure of ready El characterizes functionality, connectivity and concentration of chemical or physical knots, function of the molecular-weight distribution (MWD).

The method of NMR relaxation allows to define topological structure and molecular mobility in El. In crosslinked El at high temperatures ("a high-temperature plateau") time of spin-spin relaxation $T_{2}$ is proportional to the average molecular weight of internodal chains, the initial part of the signal of free induction decay (FID) depends on MWD, and amplitude of a final part is inversely proportional time [1].

Numerous experimental NMR data on linear flexible polymers (rubbers and thermoplastics) show that, in the amorphous state at $T>T_{g}\left(T_{g}\right.$ is the glass transition temperature), the FID is exponential for short chains with a average molecular weight $M_{w}<10^{4}$ and not exponential for longer chains $M_{w}>10^{4}$ [4]. And, irrespective of chemical structure of the polymer, the experimental dependence of the spin-spin relaxation time $T_{2}$ on the molecular weight $M_{w}$ and temperature shows that $T_{2}$ decreases with an increase in $M_{w}$ and at high temperature region has two clearly pronounced breaks [4]. The explanation of observed dependences of $T_{2}\left(M_{w}\right)$ is offered

${ }^{*}$ corresponding author; e-mail: tan@icp.ac.ru qualitatively in work [4], and connected with change of character of molecular motion with a growth $M_{w}$. However, this theory does not explain the physical nature of the breaks in the $T_{2}\left(M_{w}\right)$ curve with an increase of $M_{w}$.

Self-diffusion occurs due to random translational motions of molecules with excess kinetic energy [5]. It determines the specifics of many physicochemical processes in flexible polymers. The translational diffusion of molecules provides important information on the viscosity, intermolecular interaction, and structural organization of EL. Its intensity is quantitatively characterized by the self-diffusion coefficient $D$. The most reliable values of $D$ are obtained by the NMR pulsed magnetic field gradient stimulated echo technique (PGSE) [5], which is a direct method for measuring the spatial displacement of molecules in the direction of the applied magnetic field gradient, without any assumptions on the behavior of the autocorrelation function at long times. The numerous experimental data obtained by the PGSE method in linear flexible polymers (rubbers and thermoplastics), show that observed dependences of diffusion attenuation of a stimulated echo and mean squared displacement change a form from linear to nonlinear with growth of $M_{w}$ and coefficient of self-diffusion $D$ testifies to anomalous diffusion [5].

From experimental data $[4,5]$ it is known that the coefficient of self-diffusion depends on the average molecular weight of polymer as $D \propto M^{-\mu}$, and $\mu$ can accept values from 0.5 to 3 . Earlier we developed the theory of diffusion attenuation in the cross-linked polymers $[1,2]$ which showed that the mean squared of displacement $\left\langle S^{2}\right\rangle \log$ arithmic depends on time, and $D$ logarithmic depends on $M_{w}$. However, so far there was no the theory, allowing to explain regularities of influence of molecular weight on self-diffusion coefficient in linear polymers.

The purpose of this work is represent the theory of FID and diffusion attenuation in linear flexible polymers and an explanation of observed dependences of $T_{2}$, and coefficient of self-diffusion $D$ on the average molecular 
weight of a polymeric chain.

\section{Results and discussion}

\subsection{The Theory of free induction decay in cross-linked polymers}

The theory [1] is based on the concept of residual dipole-dipole interactions (DDI) connected with anisotropy of internodes chains, a polymer chain consists of $N_{c}$ statistically independent segments, and the motion of the polymer chain in a wide temperature range at $T>T_{g}$ is envisioned as consisting of several types of motion on disparate time scales. In the lowtemperature range, only small-scale molecular motion inside segments is manifested. In the middle-temperature range, large-scale (segmental) motion is manifested. The high-temperature range is characterized by the motion of the chain as a whole associated with all possible conformational positions of the chain segments.

In this model of the polymer chain dynamics, the molecular motion correlation function $k(\tau)$ is the next:

$$
k(\tau)=(1-\alpha) k_{1}(\tau)+\alpha k_{2}(\tau)+k_{3}\left(\theta, \mathrm{N}_{\mathrm{c}}\right),
$$

where $N_{c}$ is a chain length, equal to quantity of statistical segments, $k_{1}(\tau)$ is the Bloembergen-Purcell-Pound correlation function, which characterizes small-scale motions of the polymer chain at $T_{g}<\mathrm{T}<\left(T_{g}+50 \mathrm{~K}\right)$

$$
k_{1}(\tau)=\mathrm{e}^{-\tau / \tau_{c}},
$$

where $\tau_{c}$ is the characteristic correlation time of molecular motion, $k_{2}(\tau)$ is the Kargin-Slonimskii-Rouse correlation function, which characterizes large- scale motions of chain segments at $\left(T_{g}+50 K\right)<T<\left(T_{g}+100 K\right)$,

$$
k_{2}(\tau)=N_{0}^{-1} \sum_{p=1}^{N} \mathrm{e}^{-p^{2} \pi^{2} \tau / \tau_{c} N_{0}^{2}},
$$

$\alpha$ is a fraction of large-scale motion, the empirical coefficient (for a network structure and chains with free ends, $\alpha=0.05$ ); $N_{0}$ is the average chain length (for a polymer network, $N_{0}=N_{0 c}\left(N_{0 c}\right.$ is the average chain length between entanglements), $k_{3}\left(\theta, N_{c}\right)$ is the correlation function characterizing the motion of the chain as a whole at $T>\left(T_{g}+100 K\right)(\theta$ is the angle between the magnetic field direction and the radius vector connecting the ends of the chain),

$$
k_{3}\left(\theta, N_{c}\right)=\left(3 \cos ^{2} \theta-1\right)^{2} \ln N_{c} / N_{c}^{2} .
$$

Function $k_{3}\left(\theta, N_{c}\right)$ is determined from the FID for a separate chain [1]:

$$
g\left(N_{c}, \theta, t\right)=\exp \left[-\omega_{\text {loc }}^{2}\left(3 \cos ^{2} \theta-1\right)^{2} t^{2} \frac{\ln N_{c}}{N_{c}^{2}}\right] .
$$

Hereinafter, we use the following designations: $N=\frac{N_{c}}{\sqrt{\ln N_{c}}}, \omega_{\text {loc }}$ is a mean local field of any spin of the segment induced by spins of neighboring chain segments.

To calculate the FID for the entire sample, Eq. 6 should be averaged over angles and chain lengths between nodes
$P(N)$. For an non-orientated polymer, the distribution over angle $\theta$ is isotropic, and the FID takes the form

$$
G_{3}(t)=\int_{1}^{N} \int_{0}^{\pi / 2} g(N, \theta, t) P(N) d \cos \theta d N .
$$

The general expression of the FID in a polymer network is the next one:

$$
G_{c}(t)=G_{1}(t) G_{2}(t) G_{3}(t) .
$$

$G_{1}(t), G_{2}(t)$ values are calculated using the AndersonWeiss model with corresponding correlation functions of molecular motions $k_{i}(\tau)$ :

$$
G_{i}(t)=\exp \left(-\omega_{\text {loc }}^{2} \int_{0}^{t}(t-\tau) k_{i}(\tau) d \tau\right), i=1,2 .
$$

The distribution over chain lengths between nodes $(N)$ is an analogue of the MWD in polymer networks. It is worth noting that, at $t / N_{0 c} \gg 1$ there appears slowly decaying component of $\mathrm{G}_{c}(t)$ with the amplitude being asymptotically proportional to $N_{0 c} t^{-1}$. The major contribution to the FID is made by dipole-dipole interactions of chain spins oriented at the magic angle to the magnetic field $[1,2]$. In this case the line shape has very narrow non-Lorentzian top. Numerical calculations of FID for different $P(N)$ (Fig. 1) show that the influence of MWD appear at the beginning part of FID, the final part is independent on $P(N)$.

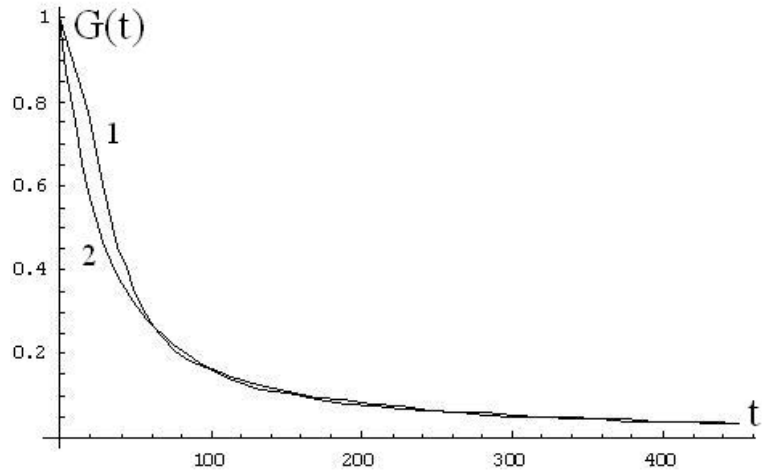

Fig. 1. The influence of MWD $P(N)$ on FID in crosslinked polymers: $P(N)$ is Gaussian $(1), P(N)$ is exponential (2).

\subsection{The theory of free induction decay in linear polymers}

For research of interrelation of average molecular weight with topological structure of linear flexible polymers we developed the theory [6] in which the following model of polymer is considered. A short-chain polymer at $M_{w}<10^{4}$ can be envisioned as a chain with free ends. An increase in $M_{w}$ leads to the appearance of physical nodes (entanglements), and, once a certain $M_{w}$ value has been achieved, there is formed a quasi-network of entanglements, which is most pronounced in the high- temperature range. In this model, the free polarization decay in linear polymers is represented as the sum of the FIDs of 
chains with free ends $\left(G_{0}(t)\right)$ and chains with fixed ends $\left(G_{c}(t)\right)$ :

$$
G(t)=(1-p) G_{0}(t)+p G_{c}(t),
$$

where $p$ is the fraction of chains connected by entanglements.

For the quasi-network of entanglement, the FID $G_{c}(t)$ is described using the previously developed theory of NMR spectra of polymer networks [6]. FID for chains with free ends $G_{0}(t)$ is calculated using the AndersonWeiss model (Eq. 8) with the molecular motions correlation function $k_{0}(\tau)$ :

$$
k_{0}(\tau)=(1-\alpha) k_{1}(\tau)+\alpha k_{2}(\tau),
$$

where the correlation functions $k_{1}(\tau)$ and $k_{2}(\tau)$ are determined by Eqs. 3 and 4, respectively. In Eq. $4, N_{0}=N_{01}$, ( $N_{01}$ is the average length of chains with free ends).

To study the effect of the average molecular weight on the topological structure of linear polymers, the FID was simulated at different average lengths of polymer chains in a wide temperature range.

Comparison with experimental data was performed taking into account that $M_{w}=m_{s} N_{0}, m_{i}$ is the molecular weight of the statistical segment, and that temperature is related to the correlation time $\tau_{c}$ by the Arrhenius law, as was demonstrated in $[1,6]$.

The FID was calculated by Eq. (9). The average chain length in the entanglement quasi-network was taken to be $100\left(N_{0}=100\right)$. In calculation of $G_{3}(t)$ by Eq. 6 , the Gaussian function of the chain length distribution $P(N)$ was taken. All calculations were performed for dimensionless time expressed in units of the local field $\omega_{\text {loc. }}$ The results of calculations with correlation functions (Eq. 1) and (Eq. 10) in the high-temperature range at $\tau_{c}=5 \times 10^{-4}$ are shown in Fig. 2. As is seen, at $N_{0}<30$ in the absence of entanglements $(p=0)$, the FID is exponential (curves 1 and 2), and the $T_{2}$ values decrease with an increase in the chain length.

At $N_{0} \geq 50$, the $p$ values were determined from the ratio $p=N_{0} / N_{0 c}$. The calculations showed that, at $p \geq 0.3$, the FID shape becomes no-exponential (curves 3 and 4) and the effect of the entanglement quasi-network manifests itself. And, it is possible to determine by NMR method existence in a sample of $10 \%$ of a physical network of entanglements.

\subsection{The theory of diffusion attenuation of stimulated echo}

According to the theory of spin echo with magnetic field gradient, the amplitude of the $i$-th echo signal $A_{i d}$ $(1 \leq i \leq 5)$ is related to the mean square of the random phase change (phase shift) $\left\langle\delta^{2} \phi_{i}\right\rangle$ due to a change in the local magnetic field on the nucleus spin as $[5,7]$ :

$$
A_{i d}=\exp \left[-\frac{1}{2}\left\langle\delta^{2} \phi_{i}\right\rangle\right] \text {. }
$$

For definition of relation $\left\langle\delta^{2} \phi_{i}\right\rangle$ with the mean square of diffusive shift of nuclear, formulas correct for any casual processes are received.

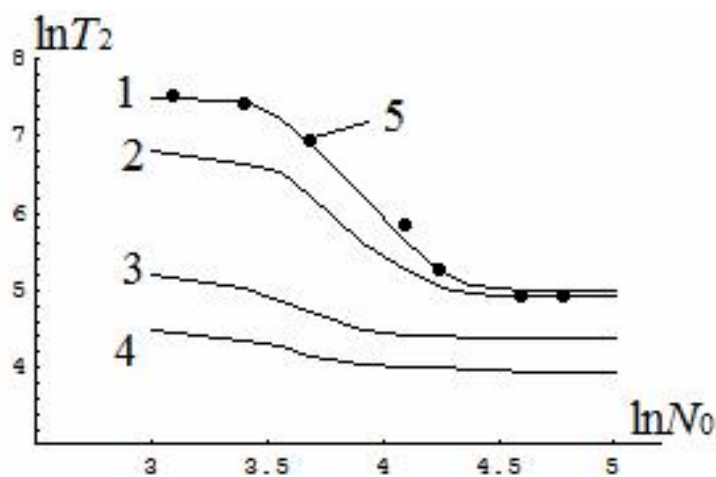

Fig. 2. Dependence of spin-spin relaxation time $T_{2}$ on the average chain length at different correlation times of molecular motion $\tau_{c}: 5 \times 10^{-4}(1), 10^{-3}(2), 5 \times 10^{-2}$ (3), $10^{-2}(4)$, experimental data points [5] (5).

Usually the signal of diffusion attenuation of stimulated echo $A_{2 d}$ is asymptotically described by the Stejskal-Tanner formula:

$$
A_{2 d}=\exp \left[-\frac{\gamma^{2} g^{2} D}{6} t_{1}^{2}\left(t_{d}-\frac{t_{1}}{3}\right)\right],
$$

where $\gamma$ is the gyromagnetic ratio for the given nucleus, $g$ is the magnetic field gradient, $t_{1}$ is the pulse duration, and $t_{d}$ the time interval between pulses.

On the basis of the general theoretical approach developed earlier $[3,7]$ the dependences of signals of diffusion attenuation and coefficient of self-diffusion of D in crosslinked and linear El with a various $M_{w}$ are investigated.

In the presence of a magnetic field gradient the mean square of the phase shift in the time interval $\left[t_{1}, t_{2}\right]$ is related to the mean squared displacement $\left\langle S^{2}(t)\right\rangle$ for the translational diffusion as

$$
\begin{aligned}
& \left\langle\delta^{2} \phi\left(t_{1}, t_{2}\right)\right\rangle=\frac{\gamma^{2} g^{2}}{3}\left(\left(t_{2}-t_{1}\right) \int_{t_{1}}^{t_{2}}\left\langle S^{2}\left(t^{\prime}\right)\right\rangle d t^{\prime}\right. \\
& \left.-\int_{0}^{t_{2}-t_{1}}\left(t_{2}-t_{1}-t^{\prime}\right)\left\langle S^{2}\left(t^{\prime}\right)\right\rangle d t^{\prime}\right) .
\end{aligned}
$$

The value of $\left\langle S^{2}(t)\right\rangle$ is set by the equation:

$$
\left\langle S^{2}(t)\right\rangle=2\left\langle V^{2}(0)\right\rangle \int_{0}^{t}\left(t-t^{\prime}\right) k\left(t^{\prime}\right) d t^{\prime}
$$

where $\left\langle V^{2}(0)\right\rangle$ is the diffusion rate variance, and $k(t)$ is the correlation function. The first term in Eq. 13 does not contribute to the echo signal, being cancelled out in the process of its formation. In this case one can consider only steady state part of phase shift. Then the diffusion attenuation of the echo signals is calculated with the help of function $F(t)$ :

$$
F(t)=\int_{0}^{t}\left(t-t^{\prime}\right)\left\langle S^{2}\left(t^{\prime}\right)\right\rangle d t^{\prime} .
$$

Thus using Eq. 11, 13, 15, we obtained the next expression for $A_{2 d}$ :

$$
A_{i d}=\exp \left[-\frac{\gamma^{2} g^{2}}{6}\left(F\left(2 t_{1}+t_{d}\right)\right.\right.
$$




$$
\left.\left.+F\left(t_{d}\right)-2 F\left(t_{1}+t_{d}\right)-2 F\left(t_{1}\right)\right)\right]
$$

In this case, the diffusion attenuation of the other spin echoes has a form similar to Eq. 16, but the observation time corresponds to the time of generation of the echo.

For sufficiently short pulse durations, the expansion of the right hand side of Eq. 15 in a series in $t_{1}$ to terms of the order of $t_{1}^{2}$ under the condition

$$
\frac{2 F\left(t_{1}\right)}{t_{1}^{2}}<<\left\langle S^{2}\left(t_{d}\right)\right\rangle
$$

the well-known formula $[5,7]$ was obtained:

$$
-\frac{6 \ln A_{2 d}}{\gamma^{2} g^{2} t_{1}^{2}}=\left\langle S^{2}\left(t_{d}\right)\right\rangle \text {. }
$$

The self-diffusion coefficient is normally determined using the condition of linear dependence of the mean square displacement on the time:

$$
\left\langle S^{2}\left(t_{d}\right)\right\rangle \sim D t_{d} .
$$

At the condition Eq. 19 the formula Eq. 18 moves to well-known Stejskal-Tanner formula Eq. 12 for the selfdiffusion coefficient $D$.

Calculations by Eq. 18 is shown that in rarely-crosslinked polymers and quasi-network of entanglements the coefficient of $D$ logarithmic depends on the average length of a chain $N_{0}$ [7].

Modeling of diffusion attenuation of a stimulated echo Eq. 16 is carried out by means of the theory [7] for crosslinked polymers with a various chains length $N_{0}$. Correlation function of molecular motions $k_{m}(t)$ can be to received from FID (Eq. 7) in the assumption that full $G(t)$ can be described also in Anderson-Wiess's model (Eq. 8). As it follows from the formula (Eq. 8), for definition of $k_{m}(t)$ it is necessary to take a negative logarithm from function $G(t)$ and twice to differentiate on $t$.

It was shown, that dependence of mean squared displacement $\left\langle S^{2}\left(t_{d}\right)\right\rangle$ on $t_{d}$ for polymer networks is non linear $\left\langle S^{2}\left(t_{d}\right)\right\rangle \ln t_{d}-\ln N_{0}$. Calculations of self-diffusion coefficient $D$ were performed with $t_{d}=80$ and $t_{d}=400$ where the plot of $\left\langle S^{2}\left(t_{d}\right)\right\rangle$ versus $t_{d}$ is conditionally linear and the condition $t / N_{0 c} \gg 1$ is fulfilled. It was shown that MWD has no effect on $D$ at $t_{d}=400$, however, at shorter $t_{d}$ times, different MWD can be observed. Calculations (Fig. $3(1)$ ) were performed in the high temperature range at $t_{d}=400$ and $7<N_{0}<177$. As is seen, $D$ decreases with an increase in $N_{0}$; as it follows from our theory $D \sim$ const $-\ln \left(N_{0}\right)$. Comparison of the calculation with experimental data on poly(ethylene glycol) at $T=373$ (Fig. 3(2)) showed their consistence. This theoretical approach has allowed explaining experimentally observed anomalous diffusion in polymeric networks. It should be noted that when studying diffusion processes in linear polymers without knots (in polymers with small length of a chain), it is necessary to consider that macromolecules can unite in "long-living" associates [3] due to inter-molecular interaction. Similar micro-heterogeneity of polymeric systems also is traced

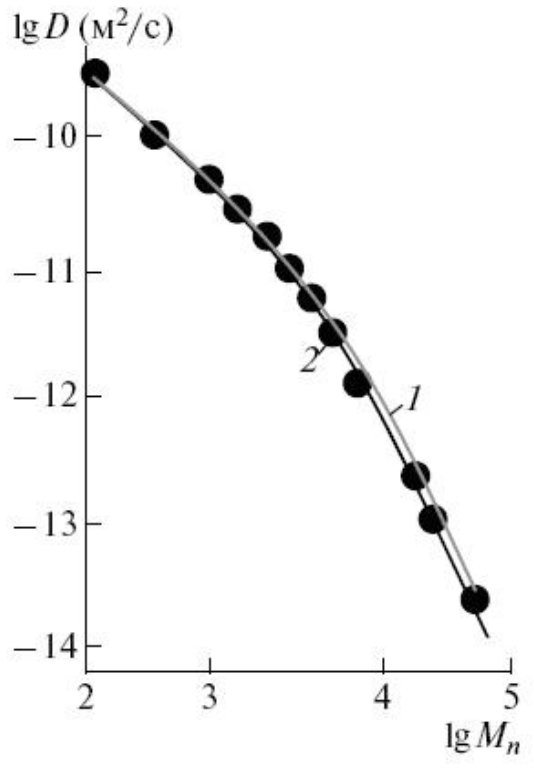

Fig. 3. Dependence of $D$ on the molecular mass $M_{n}$ at $t_{d}=400(1)$, points (2) are experimental data [5].

by the NMR method, allowing to define concentration of the associated molecules, the size of associates and time of life of the associated condition [3]. Therefore in our calculations the contribution of associates was also considered by means of the additional correlation function of small-scale molecular motion. Thus, the correlation function describing molecular motion of polymer chains without entanglements and taking into account associates looks as follows:

$$
k(\tau)=(1-\alpha) k_{1}(\tau)+\alpha k_{2}(\tau)+k_{a}(\tau),
$$

where $k_{a}(\tau)$ is Bloembergen-Purcell-Pound's correlation function.

Calculations were carried out in a high-temperature case, for calculation of $k(\tau)$ by Eq. 2, 10 time of correlation was equal to $\tau_{c}=5 \times 10^{-4}$, and $k_{a}$ values were calculated at $\tau_{c}=5 \times 10^{-3}$ as in associates characteristic time of interaction of two spins considerably exceeds interaction time of free chains. For calculation of signals of diffusion attenuation the following formula was offered:

$$
A_{2 d}\left(t_{d}\right)=(1-p) A_{2 d 0}\left(t_{d}\right)+p A_{2 d c}\left(t_{d}\right) \text {. }
$$

Here $A_{2 d 0}\left(t_{d}\right)$ is a diffusion attenuation in polymers without cross-links, $A_{2 d c}\left(t_{d}\right)$ is a diffusion attenuation in polymers with entanglements, $p$ is a part of entanglements.

Results of calculations are given in Fig. 4. Modeling showed that at any $N_{0}$ the form of diffusion attenuation is faster in emergence of entanglements (curve 1) than in absence of entanglements (curve 2) and corresponds to experiments [5]. From Fig. 4 it is visible that dependence of $D\left(N_{0}\right)$ qualitatively corresponds to dependence of $T_{2}\left(N_{0}\right)$ (Fig. 2). Thus it is possible to allocate three separate areas: on an initial site of a curve slow decrease of values of coefficient of self-diffusion (the area corresponding to chains with the free ends) is observed, on 


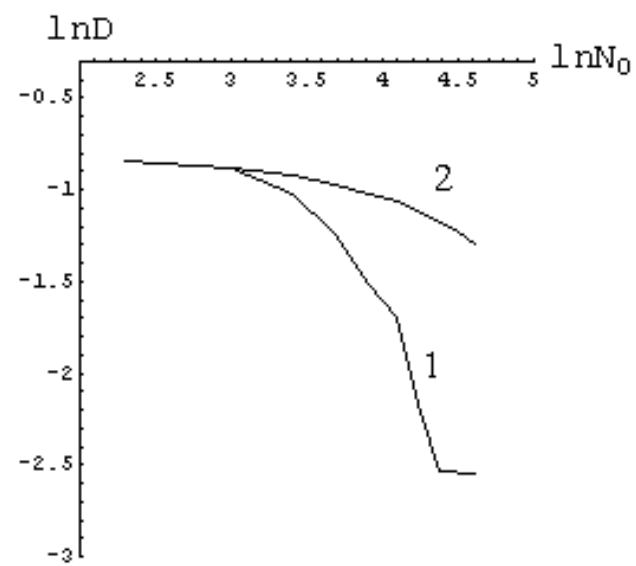

Fig. 4. Dependence of $\ln D$ on the average chain length at $t_{d}=80$ in linear polymers: polymer chains with entanglements (1) and without entanglements (2).

the second site - fast decrease of values of coefficients of self-diffusion (area of growth of a share of a physical network of entanglements), on the third site of value of coefficient the self-diffusion practically don't change with growth of chain length (area in which all polymer represents as a quasi-network of entanglements). The obtained dependence of $D\left(N_{0}\right)$ qualitatively corresponds to experiments $[4,5]$.

\section{Conclusions}

The theory and comparison with the experiments, allowing reveal legitimacy of influence of average molecular weight on topological structure and self-diffusion in elastomers are offered. Emergence of pronounced breaks in high-temperature area on the dependence of time spinspin relaxation on the average chain length is explained by change of topological structure of linear polymers and formation of a quasi-network of entanglements at average molecular mass chains $M_{w}>10^{5}$. The same structural changes influence on the dependence of self-diffusion coefficient $D$ on $M_{w}$. It allows analyze topological structure of linear polymers at $M_{w}>10^{4}$ there are chains with free end and associates, at $M_{w}>10^{4}$ the share of a physical network of entanglements growths and defines nature of anomalous diffusion. It is shown that in this case the self-diffusion coefficient logarithmic depends on $M_{w}$.

\section{References}

[1] T.P. Kulagina, V.V. Marchenkov, B.N. Provotorov, Polymer Sci. U.S.S.R. 31, 420 (1989).

[2] T.P.Kulagina, V.M.Litvinov, K.T.Summanen, J. of Polymer Sci. Part B: Polymer Physics 31, 241 (1993).

[3] T.P. Kulagina, G.E. Karnaukh, L.P. Smirnov, Doklady Phys. Chem. Part 2 421, 216 (2008).

[4] M. Kehr, N. Fatkullin, R. Kimmich, J. Chem. Phys. 126, 094903 (2007).

[5] A.I. Maklakov, V.D. Skirda, N.F. Fatkullin, Samodiffuziya $v$ rastvorakh i rasplavakh (Self-Diffusion in Solutions and Melts). Kazan Uni., Kazan, 1987.
[6] T.P. Kulagina, V.A. Varakina, A.N. Kuzina, Doklady Phys. Chem. Part 2 444, 79 (2012).

[7] T.P. Kulagina, G.E. Karnaukh, A.N. Kuzina, L.P. Smirnov, Russian Journal of Phys. Chem. B 7, 170 (2013) 